\title{
The paralogous gene of myostatin deficiency does not improve the growth of Red seabream (Pagrus major)
}

\author{
Mitsuki Ohama1, Kenta Kishimoto², Masato Kinoshita², Keitaro Kato' ${ }^{1}$, Youhei Washio1* \\ ${ }^{1}$ Aquaculture Research Institute, Kindai University, Shirahama 3153, Nishimuro, Wakayama 649-2211, Japan. \\ 2 Division of Applied Bioscience, Graduate School of Agriculture, Kyoto University, Kitashirakawa-Oiwake, \\ Sakyo-ku, Kyoto, 606-8502, Japan.
}

${ }^{*}$ Corresponding author: washio@kindai.ac.jp

\begin{abstract}
To improve livestock and aquaculture-raised fish as food, targeted mutagenesis using genome editing technologies is becoming more realizable. Myostatin (Mstn), which functions as the negative regulator of skeletal muscle growth, is one of the major targets to improve the edible ratio of livestock and farmed fish. We previously reported that the deficiency of Pm-mstn, one of Myostatin paralogs, improves muscle growth and changes body shape in a finfish species, red seabream (Pagrus major), as a result of editing the gene by means of CRISPR/Cas9. In this study, we established Pm-mstnb-deficient red seabream, which is a null-allelic mutant of another paralogous gene of Myostatin in the species, and analyzed their phenotype in terms of growth traits and body shape. A comparison of all growth traits between Pm-mstnb ${ }^{w t / w t}$ and Pm-mstn $b^{-5 /-5}$ revealed no significant differences. In addition, all metrics for body shape, defined as the ratios of body depth, body width, and depth of the caudal peduncle to body length, respectively, were also similar in Pm-mstn $b^{w t / w t}$ and Pm-mstn $b^{-5 /-5}$. Therefore, we concluded that $P m$ $m s t n b$ does not function as a negative regulator of skeletal muscle growth in red seabream.
\end{abstract}

Keywords: Red seabream, CRISPR/Cas9, Myostatin, Muscle, Growth, Body shape

\section{Introduction}

Red seabream (Pagrus major) (Temminck et Schlegel,1843) is one of the most commercially important species for fisheries and aquaculture in East Asia. For aquaculture, a fast-growing strain of red seabream had been produced by means of selective breeding in Japan, so the productivity of red seabream culturing had been improved (Murata et al., 1996). While the mass production became better, the edible ratio of red seabream as fillets (edible parts) is almost less than $40 \%$ to its total body weight, no matter whether the fish is cultured or wild-caught. The other $60 \%$ such as head, skins, bones, and internal organs are wasted during processing. Therefore, we have been aiming to improve the processing yield by increasing the edible part of red seabream by breeding technologies.

It is a problem that the classical selective breeding of spontaneous mutants requires a long-time period to produce a brand-new breed, due to the randomness of natural mutation and the generation time of target fish. Recently, genome editing technologies have become an increasingly feasible means to produce useful organisms as a rapidbreeding technology (Xiong et al., 2015; Tait-Burkard et al., 2018; Gratacap et al., 2019). To accelerate finfish breeding, we had demonstrated that the efficient method for inviting genome editing tools to marine aquaculture fish eggs (Kishimoto et al., 2019), and an example of the establishment of a new breed of homozygous gene disrupted red seabream within a few years (Kishimoto et al., 2018).
In a previous report, using CRISPR/Cas9 (Clustered Regularly Interspaced Short Palindromic Repeats / CRISPR associated nuclease 9) systems, we demonstrated that knocking out the gene Pm-mstn increases skeletal muscle mass and changes body shape in red seabream compared to Pm-mstn ${ }^{w t / w t}$ (Kishimoto et al., 2018). Pm-mstn is a paralogous gene of Myostatin, which is the negative regulator of skeletal muscle mass, and a member of the transforming growth factor $\beta$ superfamily (TGF- $\beta$ ). At that time, we had also produced another Myostatin paralogdisrupted breed, a Pm-mstnb-disrupted line, but had not yet determined whether this line differed in growth traits or body shape compared to Pm-mstnb ${ }^{w t / w t}$ fish, other than at generation 0 (Go) (Kishimoto et al., 2018). We had shown that there was no difference in body mass index between highly Pm-mstnb-mutated Go fish and Pm-mstn $b^{w t / w t}$ fish (Kishimoto et al., 2018). Because Go fish that are produced by microinjecting genome editing tools into fertilized eggs (as in this study, in which we have used wild-type eggs and semen) often show genetically mosaic-bodies on a cell by cell basis, the effect of gene disruption is not always apparent in Go (Brocal et al., 2016; Mehravar et al., 2019). While CRISPR targets a specific region of a specific gene, the deletions it introduces can vary in location and length, so it can produce multiple mutant genotypes. During the cleavage of a genome editing tools-injected cell, the targeted DNA sequences in each individual cell of the embryo are mutated separately and this produces varied genotypes; consequently, cells with different mutations can be present in the same $\mathrm{G}_{0}$ organism. There was a possibility that the fish with genetically mosaicbodies might be either phenotypically intermediate or phenotypically the same as the wild type, because intact cells 
and cells with in-frame mutations might mask the effect of the deleterious mutation. Thus, the effects of gene editing are not always apparent in the $\mathrm{G}_{0}$ generation. It is therefore important to examine the effects of gene-editing technologies in the $\mathrm{F}_{1}$ generation.

Here, we aim to elucidate the function of Mstnb in red seabream and the potential for gene-editing targeting this gene to improve growth in this important aquaculture species. We have focused on the phenotype of the homozygous Pm-mstnb deficient fish of the $\mathrm{F}_{1}$ generation compared with Pm-mstnb ${ }^{w t / w t}$ fish, in terms of their growth traits and body shape.

\section{Materials and Methods}

\section{Ethics statement}

The experiments in this study were performed in accordance with the Guidelines for Animal Experimentation at the Aquaculture Research Institute, Kindai University. The fish handling and sampling methods were approved by the committee in Aquaculture Research Institute, Kindai University (2014-2019). All efforts were made to minimize fish suffering.

\section{Experimental fish}

All fish were kept at the Shirahama station, Aquaculture Research Institute Kindai University, Japan. The Pm-mstnbedited red seabream had been created utilizing zygote injection of the CRISPR/Cas9 reagents, meaning Cas9 RNA and the Pm-mstnb-targeted single guided RNA (target DNA sequence: 5'- CCG GGA CAT GAT CCG ACA GCT GC -3') (Figure 1) (Kishimoto et al., 2018). The details of genome editing and phylogenic/syntenic relationship between Pm-mstn and Pm$m s t n b$ were shown in the previous report (Kishimoto et al., 2018). These generation $0\left(\mathrm{G}_{0}\right)$ fish were reared to 2-years old, and then 6 male fish and 3 female fish were fin-clipped and accommodated in a black polyethylene tank $(1,000$ litter, L), as the broodstocks for this study. On evenings from April to May of 2017, the bloodstocks showed random mating and natural spawning in the tank. The fertilized eggs were obtained from the drain of broodstock tank and were kept in 1 to $3 \mathrm{~L}$ poly beakers containing UV-sterilized seawater at $20^{\circ} \mathrm{C}$ until they hatched. Hatched larvae were accommodated in separate rearing tanks (200 L polycarbonate) by fertilization date as test-tanks 1 to 3 . These first filial generation $\left(F_{1}\right)$ fish were reared and fed 1 to 3 times per day with the feed-organisms and feed-formulas shown below; 6$30 \mathrm{dpf}$ (days post fertilization): docosahexaenoic acid (DHA)enriched rotifer (Branchionus plicatilis sp. complex), 23-43 dpf: DHA-enriched brine shrimp (Artemia salina nauplii), after $23 \mathrm{dpf}$ : commercial formulas depending on the size of fish mouth (new ARTECK K-2 for 23-35 dpf larvae, Marubeni Nisshin Feed, Japan) (Ambrose 600 for 30-45 dpf juveniles, and Ambrose 800 for 40-60 dpf, FEED ONE, Japan) (Pia gold 0 for 60-100 dpf, Pia gold 1 for 100-150 dpf, Pia gold 2 for 150$200 \mathrm{dpf}$, Pia gold 3 for over $200 \mathrm{dpf}$ young fish, Marubeni Nisshin Feed, Japan). For the DHA enrichment of the feedorganisms, powder of Schizochytrium sp. (Bio-Chromis, Chlorella Industry, Japan) was used. During their larval stage
(6-30 dpf), living Nannochloropsis sp. was supplemented into test-tanks to maintain water quality and to enhance the nutrient quality of the rotifers (Okauchi, 2004). Another Pm$m s t n b^{w t / w t}$ fish were spawned from the wild type-broodstocks as the control group; these were reared by the same methods in separate tanks ( $200 \mathrm{~L}$ polycarbonate) by date fertilized as the control tanks 1 and 2. At $155 \mathrm{dpf}$, passive-integratedtransponder tags (BIOMARK, HPT9, USA) were inserted into all $\mathrm{F}_{1}$ individuals for identification. At the same time, part of the fin was clipped from every fish and was kept into $100 \%$ EtOH at $-20^{\circ} \mathrm{C}$ until DNA extraction.

\section{Genotyping}

In the case of $\mathrm{G}_{0}$, fins were rehydrated, cut into $1 \mathrm{~mm}^{2}$ pieces, and then were immersed into a microcentrifuge tube with an extraction buffer consisting of $2 \mu \mathrm{L}$ Proteinase K (TaKaRa, 9034, Japan) and $98 \mu \mathrm{L}$ phosphate-buffered saline (pH 7.2). The microcentrifuge tubes containing the fin were heated at $55^{\circ} \mathrm{C}$ for 2 hours to dissolve the tissue and then heated at $98^{\circ} \mathrm{C}$ for 10 minutes to inactivate the Proteinase K. After centrifugation $(3000 \times \mathrm{g}, 1$ minute), the supernatants were recovered as DNA solutions. The PCR reaction mixture was prepared as follows: extracted DNA solution $1.0 \mu \mathrm{L}$, each primer $2.0 \mu \mathrm{L}(1.0 \mu \mathrm{M})$, distilled water $3.2 \mu \mathrm{L}$ and PCR reagents (KOD FX: $0.2 \mu \mathrm{L}, 2 \times$ PCR buffer for KOD FX: $10 \mu \mathrm{L}, 2$ mM dNTPs: $1.6 \mu \mathrm{L}$ ) (TOYOBO, KFX-101, Japan). PCR was performed according to the following conditions: $94^{\circ} \mathrm{C}$ for 2 minutes, $\left(98^{\circ} \mathrm{C}\right.$ for 15 seconds, $55^{\circ} \mathrm{C}$ for 45 seconds, $68^{\circ} \mathrm{C}$ for 1 minute) $\times 35$ cycles, then $68^{\circ} \mathrm{C}$ for 1 minute. Primer sequences for detection of mstnb-mutation were: RSB_mstnb_Fw1 (5'-CAA GCA GAT GAG GCT CCA CAG-3'), RSB_mstnb_Rv2 (5'-GCT TGG TGG CCA TGG TGA TG-3') (Fig. 1). The wild-type fish amplicon was $211 \mathrm{bp}$. To determine the mosaicism of $\mathrm{G}_{0}$, these amplicons were deep sequenced with Miseq (Illumina, USA) and KAPA Hyper Prep Kits (Roche Sequencing, 7962312001, USA) in accordance with a previously reported method (Brocal et al., 2016). After assembling the $150 \mathrm{bp}$ paired-end reads, the assembled reads were binned at the unique sequence level. We checked the frequencies of each unique sequence as a fraction of total reads per sample using PEAR: Paired-End reAd mergeR 0.9.8 (https://cme.h-its.org/exelixis/web/software/pear/index. html) and Excel 2016 (Microsoft, Japan). The three major patterns of mutation, other in-frame mutations, and other frame-shift mutations (i.e., not only deletion, but also insertion, conversion, and combinations of these) in the soma of broodstocks were listed for each individual. At that time, each rare sequence, which was defined as under $1 \%$ of total reads in a sample and assumed to be a product of PCR error, was bundled. Three wild-type fish were also deep-sequenced as the control.

In the case of $F_{1}$, after DNA extraction and PCR were performed with the same methods used for $\mathrm{G}_{0}$, a heteroduplex mobility assay (HMA) was performed with the apparatus protocol (MultiNA, Shimadzu, Japan) and DNA-500 Kit (S292-27910-91, Shimadzu, Japan) following the report (Ansai and Kinoshita, 2014). After pre-screening, representative samples were separately Sanger-sequenced and the genotype was determined. 


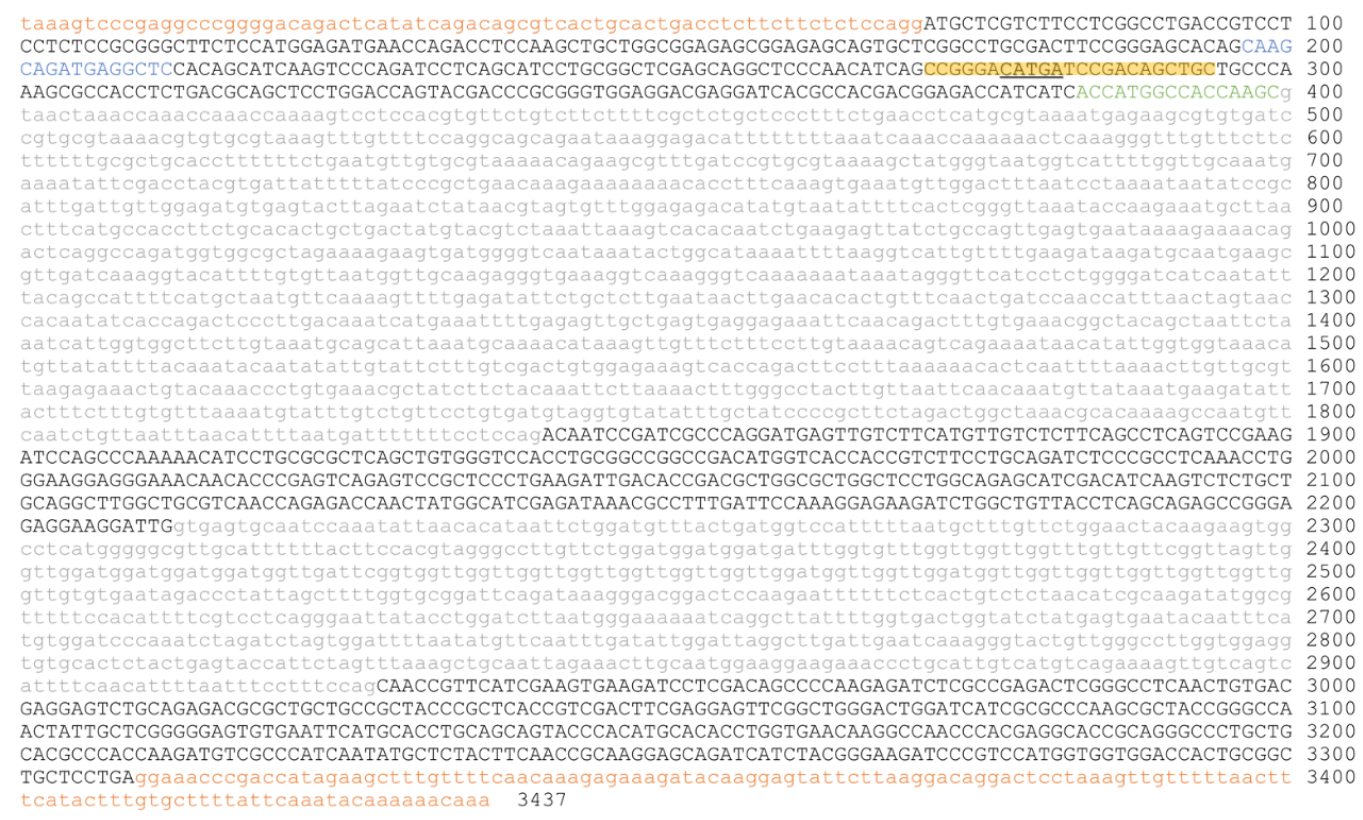

Figure 1. Presumed genomic sequence of Pm-mstnb, Pm-mstnb gene is presumed as 3 exons (black upper cases, total coding sequence are $1155 \mathrm{bp}$ ), 2 introns (gray lower cases), and untranslated region (orange lower cases). Blue and green letters show PCR primer binding sites. Yellow highlight indicates the sequence designed for the single guided RNA (reverse stranded). The represented deletion site (c. 207_211del5) is underlined.

\section{Estimation of amino acid sequences and protein domain architectures}

The genomic sequence of Pm-mstnb is shown in Fig. 1. DNA sequences of each sample were converted to their predicted amino acid sequence using software (ApE: A plasmid Editor, http://life-science-project.com/266/). To explore the domain architecture of the protein, the web-based portal (SMART: Simple Modular Architecture Research Tool, http://smart.embl-heidelberg.de/) was used.

\section{Growth traits}

For the within-tank investigation, the measurements were carried out on a tank-by-tank basis. The number of Pm$m s t n b^{w t / w t}$ and Pm-mstn $b^{-5 /-5}$ fish, respectively, in each tank and the date of measuring were as follows: tank 1 (231 dpf), 2 and 4 fish; tank 2 (229 dpf), 1 and 10 fish; tank 3 (227 dpf), 1 and 6 fish. These fish were accommodated upon hatching following the spontaneous spawning of $\mathrm{G}_{0}$ and were maintained in same tank throughout the experiment. For the between-tank investigation, the wild-type fish in control tank 1 were also measured at $230 \mathrm{dpf}$ and were compared to that of Pm-mstnb $b^{-5 /-5}$ in test tank 2(229 dpf). Measured growth traits were fork length (FL, the length from the tip of snout with closed mouth to the center of the fork in the caudal fin), standard length (SL), body width (Wi) and body weight (BW). The formula of the condition factor was BW $\times 10^{3} /$ (FL or $\mathrm{SL})^{3}$.

\section{Body Shape}

To compare Pm-mstn $b^{-5 /-5}$ fish with the body shape of Pm$m s t n b^{w t / w t}$ fish, 5 mutants (264 dpf, from test tank 3) and 5 wild-type fish (273 dpf, from control tank 2) were selected using FL size as an index, their SL and Wi were measured, and then they were photographed. From the photographs, body depth (BD) and depth of caudal peduncle (DCP) were measured using image analysis software FIJI (NIH, Append version of ImageJ software, https://fiji.sc/). The ratio of each value to the SL was calculated.

We should note that an untimely mechanical failure with water flow in test tanks 1 and 2 and control tank 1 occurred prior to the collection of body-shape data, resulting in fish mortality in those tanks and, thus, constraining our body-shape data collection to the remaining tanks (i.e. test tank 3 and control tank 2).

\section{Statistical analysis}

Mann-Whitney's U test was used to compare the body shape and growth of Pm-mstn $b^{-5 /-5}$ fish and Pm-mstn $b^{w t} / w t$ fish. For the significant difference test, the statistical processing site MEPHAS (http://www.gen-info.osaka-u.ac.jp/MEPHAS/) was used.

\section{Drawing figures and tables}

PowerPoint 2016 (Microsoft, Japan) and Excel 2016 were used for drawing figures and tables.

\section{Results}

\section{Somatic genotypes of broodstocks}

Deep sequencing revealed the spectrum of somatic mutations in broodstocks (Fig. 2). The total assembled reads were 91,713. The mean number of assembled reads in a sample (the depth of reads per sample) and the standard deviation (SD) were 7,342.8 and 3,782.7, respectively. The mutation rates (mutated reads per all reads in a fish) in each soma of $\mathrm{G}_{0}$ were generally high (Mean: $82.2 \%$, SD: 7.4). The 
soma of gene-edited $\mathrm{G}_{0}$ fish exhibited genetic mosaicism. The number of sequence variants in a single $\mathrm{G}_{0}$ fish, excluding rare variants that comprised less than $1 \%$ of reads per fish, ranged from 3 to 15 (Mean: 7.44, SD: 3.53). The percentage of rare sequence variants and/or results of amplification errors were below; $\mathrm{G}_{0}$ fish (Mean: $13.1 \%$, SD: 3.76), control fish (Mean: 17.2\%, SD: 1.16). Although there was individual variation in genetic mosaicism, the three major mutation patterns observed among $\mathrm{G}_{0}$ were as follows: a deletion of 5 nucleotides between nucleotide positions (see Fig. 1) 207 and 211 (c. 207_211del5), a deletion of 1 nucleotide at position 209 (c. 209del1) and a deletion of 2 nucleotides at positions 207-208. (c. 207_208del2) (Figure 2).

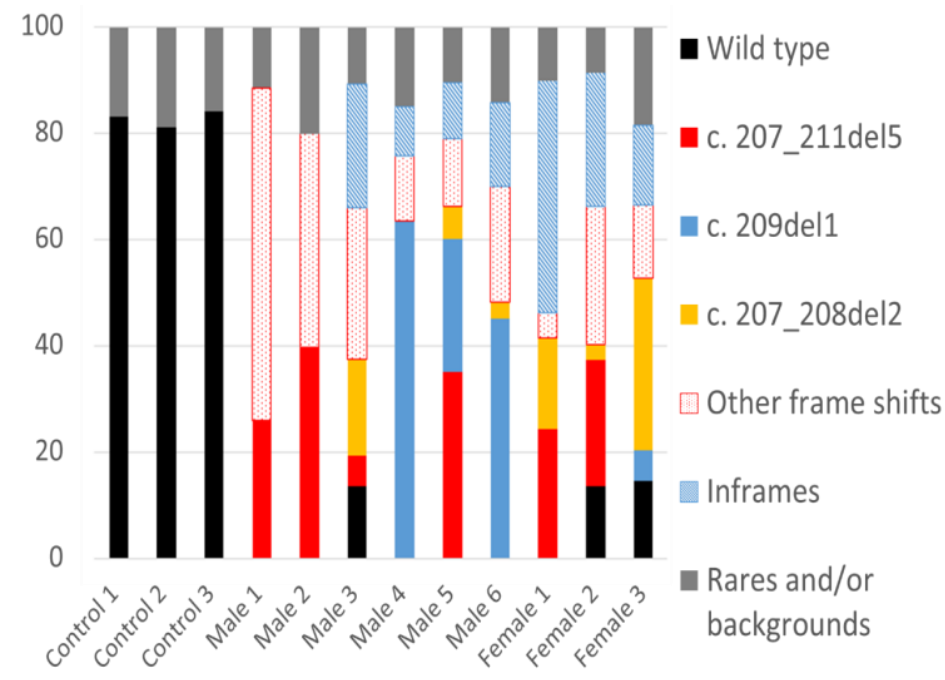

Figure 2. The spectrum of somatic mutation in the broodstocks. Columns represent the proportion of observed somatic mutations to the Pm-mstnb gene observed in each gene-edited broodstock individual. Three wild-type fish as the control (not sexed), $6 \mathrm{G}_{0}$ male, and $3 \mathrm{G}_{0}$ female were analyzed. The major variants were wild type (black), c. 207_211del5 (red), c. 209del1 (blue), and c. 207_208del2 (yellow). Other mutations shown include other frame-shifted variants (red dots), in-frame variants (blue lines), and rare variants and/or technical backgrounds (gray).

\section{Founder 1, genotypes and amino acids alteration}

The total number of $\mathrm{F}_{1}$ fish produced from Pm-mstnb disrupted $\mathrm{G}_{0}$ parents was 395 fish at $155 \mathrm{dpf}$. Within these $395 \mathrm{~F}_{1}$ fish, there were 4 homozygotes for the wild-type gene (wt/wt) offspring, 34 homozygous mutants (c. 207_211del5) $(-5 /-5), 81$ heterozygous mutants $(-5 / \mathrm{wt})$ and 276 other mutants. These remaining 276 mutants showed other variants such as (c. 209del1), (c. 207_208del2) and (c. 201_210del10) or compound heterozygous mutants. In the $\mathrm{F}_{1}$, the predominant pattern of the mutation in overall tanks was the above-mentioned 5 base deletion in c. 207211 ; therefore, we treated the fish with these mutation as the representative mutants for subsequent tests.

The homozygous 5 base deletion was assumed to cause a frame-shift in the coding sequence of Pm-mstnb and deficiency of Mstnb-protein functions. Because of that, the presumed amino acid sequence of $m s t n b^{-5 /-5}$ ends at $67^{\text {th }}$ amino acid residue and the functional domain of Mstnbprotein and the $\mathrm{C}$-terminal signal peptide region are not synthesized (Figure 3, green letters).

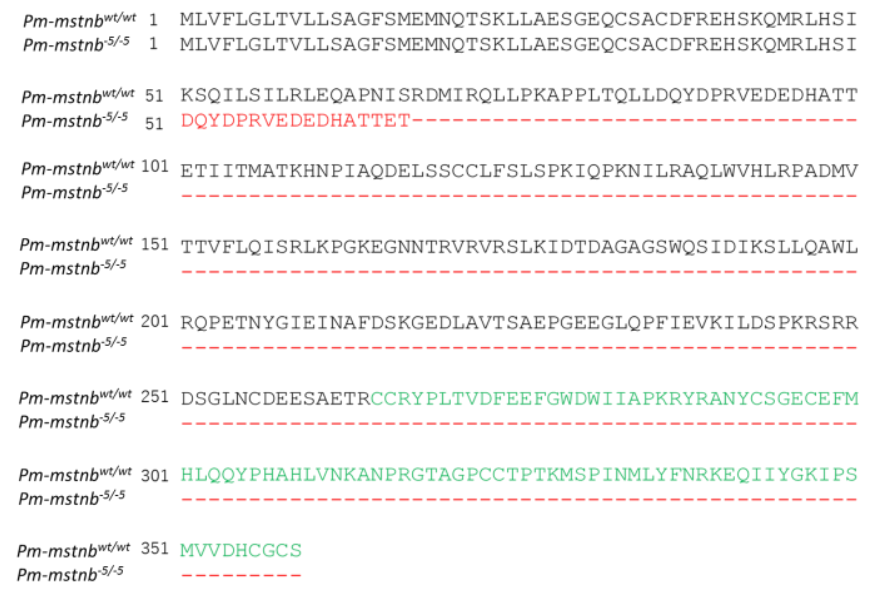

Figure 3. Alignment of presumed amino acids between $\mathrm{Pm}$ $m s t n b^{w t} / w t$ and Pm-mstn $b^{-5 /-5}$. Black and red letters indicate the homologous sequence and the nonhomologous sequence between Pm-mstn $b^{w t / w t}$ and Pm-mstn $b^{-5 /-5}$, respectively. Pm-mstn $b^{w t} / w t$ harbors 359 amino acids with a complete $C$-terminal active domain (green letters). On the other hand, $\mathrm{Pm}$ $m s t n b^{-5 /-5}$ harbors only 67 amino acids.

\section{Growth traits}

First, to exclude environmental differences between rearing tanks, we compared the growth traits between $P m-m s t n b^{w t / w t}$ and $P m-m s t n b^{-5 /-5}$ within each individual tank that had been used for rearing $F_{1}$ fish from hatching to measuring (Fig. 4, within-tank comparison). Because the numbers of Pm$m s t n b^{w t} / w t$ in each test tank were not sufficient (1 to 2 fish in each tank), statistical comparisons of Pm-mstn $b^{w t} / w t$ and Pm$m s t n b^{-5 /-5}$ within the same tank could not be performed. In general, among Pm-mstn $b^{-5 /-5}$ and Pm-mstnb ${ }^{w t / w t} \mathrm{~F}_{1}$ fish reared in the same tank, almost all values for Pm-mstn $b^{w t / w t}$ growth traits fell within the range of those for Pm-mstn $b^{-5 /-5}$ fish, with only a few values occurring slightly outside of this range (Figure 4). 

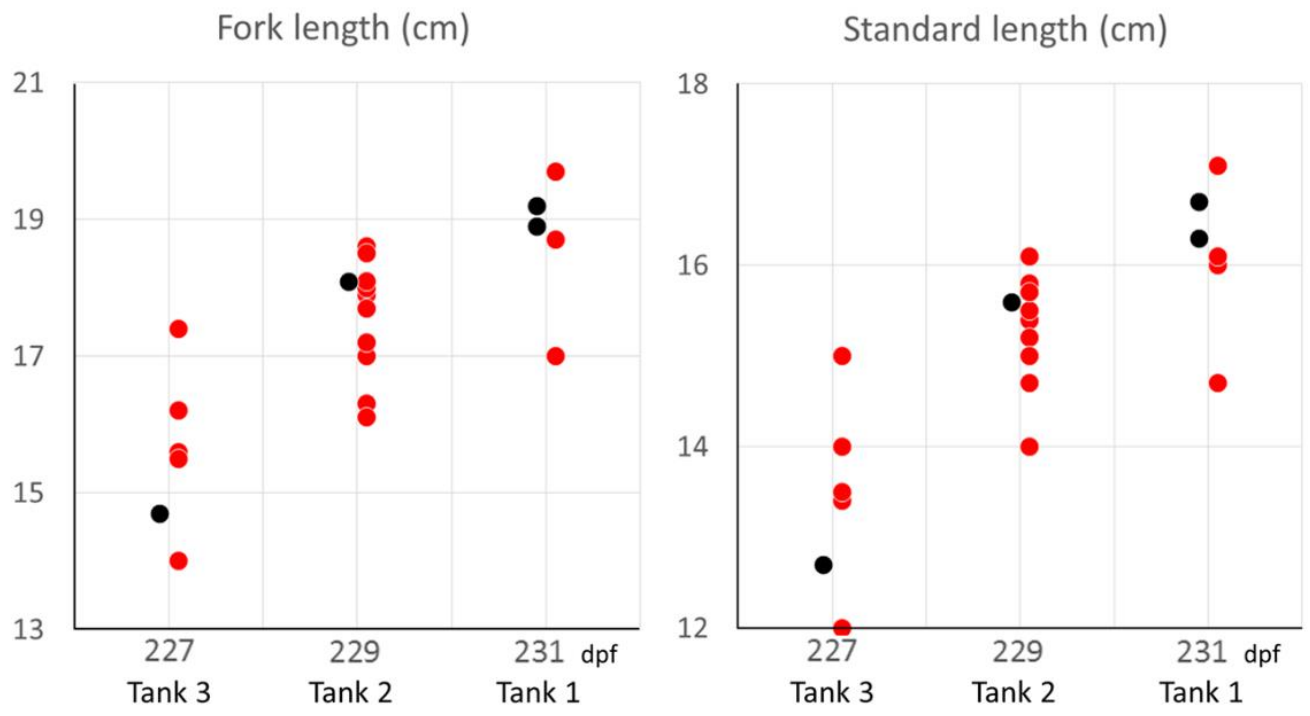

Body width (cm)

Body weight (g)
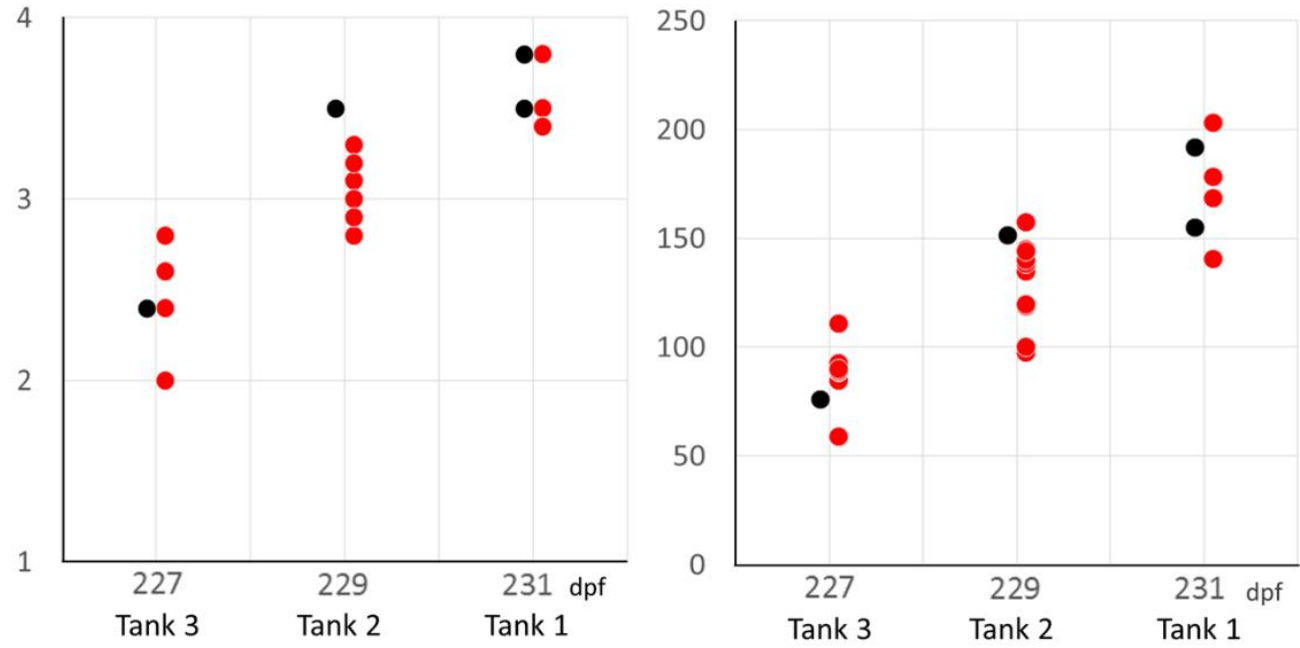

Condition factor $\left(\mathrm{BW} \times 10^{3} / \mathrm{FL}^{3}\right)$

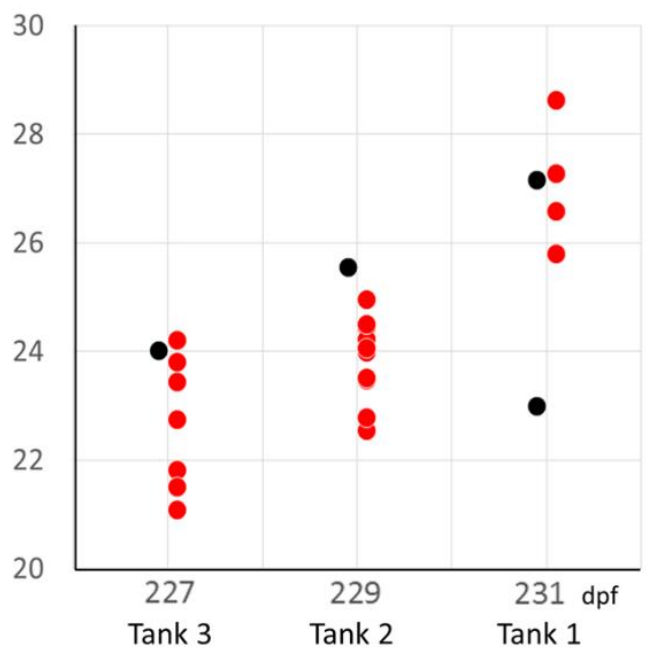

Condition factor $\left(\mathrm{BW} \times 10^{3} / \mathrm{SL}^{3}\right)$

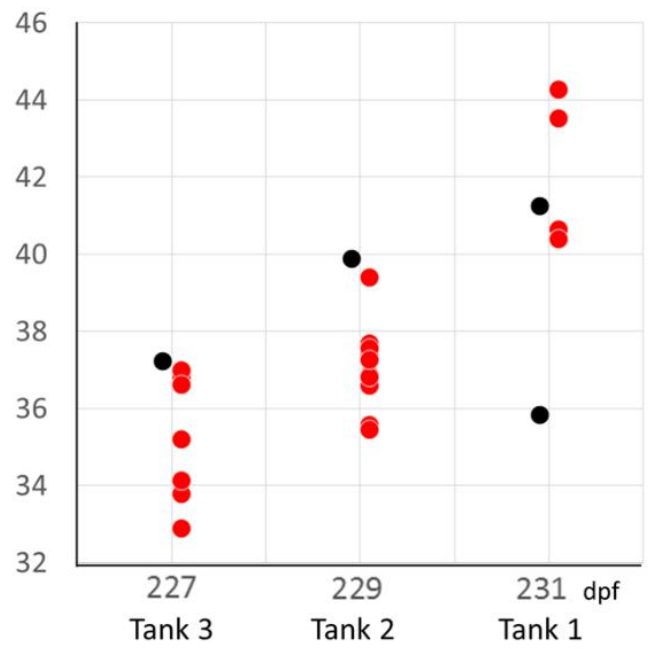

Figure 4. Growth traits of wild type and mutant fish in each test tank. Evaluated traits, days post fertilization (dpf), and names of tanks are as shown in the graphs. Black and red plots show the value of Pm-mstnb ${ }^{w t / w t}$ and Pm-mstn $b^{-5 /-5}$, respectively. 
To further examine whether the growth of Pm-mstn $b^{-5 /-5}$ differed from that of Pm-mstn $b^{w t / w t}$, we compared $10 \mathrm{Pm}$ $m s t n b^{-5 /-5}$ fish from tank 2 (229 dpf) with the same number of Pm-mstn $b^{w t / w t}$ control group fish (230 dpf) from control tank 1, because the birth dates, density of fish in each tank, and the fish condition of two groups were similar. For this between-tank comparison, there were no significant differences in any growth trait or in condition factors between the two groups $(P>0.05)$ (Mann-Whitney's U test) (Table 1).

Table 1. Growth traits of wild type and mutant fish in separated tanks.

\begin{tabular}{|c|c|c|c|c|c|c|c|}
\hline \multirow{2}{*}{$\begin{array}{l}\text { Traits or ratio } \\
\text { Fork length } \\
\text { (FL) }\end{array}$} & \multirow{2}{*}{$\begin{array}{l}\text { Unit } \\
(\mathrm{cm})\end{array}$} & \multicolumn{3}{|c|}{$\begin{array}{l}\text { Pm-mstnb }{ }^{w t} / w t \\
\text { Control tank } 1 . \\
230 \text { dpf. } \mathrm{N}=10 .\end{array}$} & \multicolumn{3}{|c|}{$\begin{array}{c}\text { Pm-mstn } b^{-5 /-5} \\
\text { Test tank } 2.229 \\
\text { dpf. } \mathrm{N}=10 .\end{array}$} \\
\hline & & 18.1 & \pm & 0.87 & 17.5 & \pm & 0.87 \\
\hline $\begin{array}{l}\text { Standard } \\
\text { length (SL) }\end{array}$ & $(\mathrm{cm})$ & 15.6 & \pm & 0.49 & 15.1 & \pm & 0.72 \\
\hline Body width & $(\mathrm{cm})$ & 3.0 & \pm & 0.10 & 3.0 & \pm & 0.15 \\
\hline $\begin{array}{l}\text { Body weight } \\
\text { (BW) }\end{array}$ & (g) & 141.3 & \pm & 20.25 & 129.6 & \pm & 19.78 \\
\hline $\begin{array}{l}\text { Condition } \\
\text { factor -FL }\end{array}$ & $\begin{array}{l}(\mathrm{BW} \\
\times 10^{3} / \\
\left.\mathrm{FL}^{3}\right)\end{array}$ & 23.5 & \pm & 1.23 & 23.8 & \pm & 0.77 \\
\hline $\begin{array}{l}\text { Condition } \\
\text { factor -SL }\end{array}$ & $\begin{array}{c}(\mathrm{BW} \\
\times 10^{3} / \\
\left.\mathrm{SL}^{3}\right) \\
\end{array}$ & 37.2 & \pm & 2.74 & 37.1 & \pm & 1.13 \\
\hline
\end{tabular}

Mean \pm Standard deviation. dpf: days post fertilization. $\mathrm{N}$ : number of specimens.

\section{Body shape}

The appearances of Pm-mstnb ${ }^{w t / w t}$ and Pm-mstn $b^{-5 /-5}$ were shown in Figure 5. The differences in FL, SL, He, Wi, DCP, BW, and condition factors between those two genotypes were not statistically different $(P>0.05)$ (Mann-Whitney's U test) (Table 2). Also, calculated ratios of BD/SL, Wi/SL and DCP/SL also showed no significant differences between $\mathrm{Pm}$ $m s t n b^{w t / w t}$ and $P m-m s t n b^{-5 /-5}(P>0.05)$ (Mann-Whitney's U test) (Table 2).

We note that the growth of the fish selected for body shape analysis from test tank 3 and control tank 2 was slower than that of the fish in the other tanks (Table 1 and 2 ). For example, the mean of FL in the control tank 1 was $18.1 \mathrm{~cm}$ at $230 \mathrm{dpf}$, while those of control tank 2 and test tank 3 were older but smaller, as were $15.5 \mathrm{~cm}$ ( $273 \mathrm{dpf}$ ) and $15.6 \mathrm{~cm}$ (264 dpf), respectively (Table 1 and 2). This might be due to the differences in rearing environments and/or the differences in the hatching date between tanks.
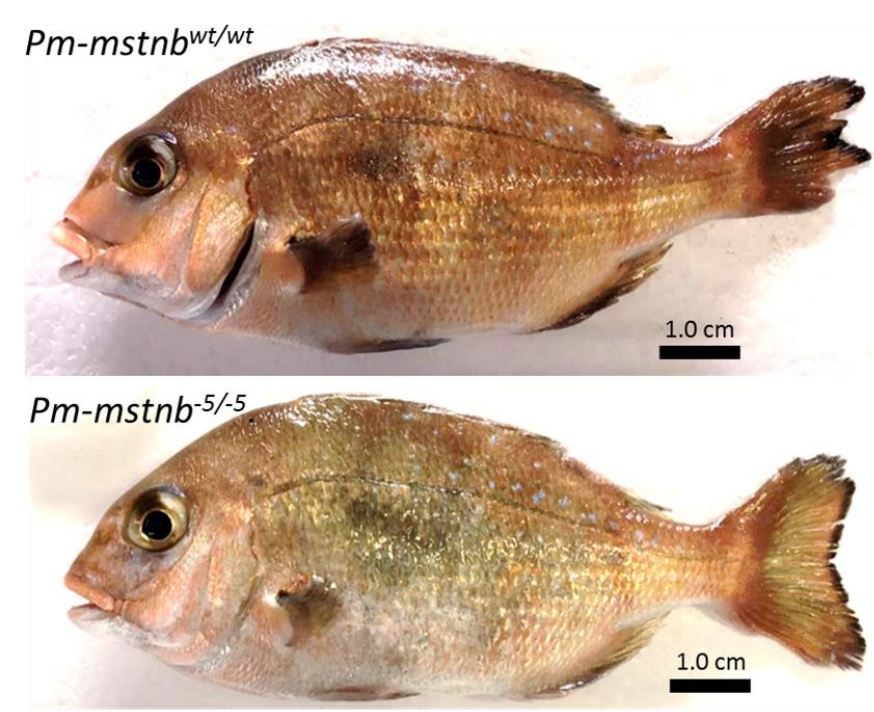

Figure 5. Appearances of wild type and mutant fish. Pm$m s t n b^{w t / w t}$ and Pm-mstn $b^{-5 /-5}$ individuals were selected from control tank 2 (273 dpf: days post fertilization) and test tank 3 (264 dpf), respectively. Bars indicate the scale as $1.0 \mathrm{~cm}$. See also Table 2 .

Table 2. Body shapes of wild type and mutant fish in separated tanks.

\begin{tabular}{|c|c|c|c|c|c|c|c|}
\hline \multirow{2}{*}{$\begin{array}{l}\text { Traits or ratio } \\
\text { Fork length } \\
\text { (FL) }\end{array}$} & \multirow{2}{*}{$\begin{array}{l}\text { Unit } \\
(\mathrm{cm})\end{array}$} & \multicolumn{3}{|c|}{$\begin{array}{c}\text { Pm-mstnb } b^{w t / w t} \\
\text { Control tank } 2.273 \\
\text { dpf. } \mathrm{N}=5 .\end{array}$} & \multicolumn{3}{|c|}{$\begin{array}{l}P m-m s t n b^{-5 /-5} \\
\text { Test tank } 3 . \\
264 \text { dpf. } \mathrm{N}=5 .\end{array}$} \\
\hline & & 15.5 & \pm & 0.60 & 15.6 & \pm & 0.70 \\
\hline $\begin{array}{l}\text { Standard } \\
\text { length (SL) }\end{array}$ & $(\mathrm{cm})$ & 13.3 & \pm & 0.52 & 13.4 & \pm & 0.62 \\
\hline $\begin{array}{l}\text { Body width } \\
\text { (Wi) }\end{array}$ & $(\mathrm{cm})$ & 2.4 & \pm & 0.19 & 2.4 & \pm & 0.19 \\
\hline $\begin{array}{l}\text { Body depth } \\
\text { (BD) }\end{array}$ & $(\mathrm{cm})$ & 6.2 & \pm & 0.22 & 6.3 & \pm & 0.39 \\
\hline $\begin{array}{l}\text { Depth of } \\
\text { caudal } \\
\text { peduncle } \\
\text { (DCP) }\end{array}$ & $(\mathrm{cm})$ & 1.6 & \pm & 0.06 & 1.6 & \pm & 0.11 \\
\hline $\begin{array}{l}\text { Body weight } \\
\text { (BW) }\end{array}$ & (g) & 79.4 & \pm & & 81.1 & \pm & 11.42 \\
\hline $\begin{array}{l}\text { Condition } \\
\text { factor -FL }\end{array}$ & $\begin{array}{c}(\mathrm{BW} \\
\times 10^{3} / \\
\left.\mathrm{FL}^{3}\right)\end{array}$ & 21.1 & \pm & 0.25 & 21.3 & \pm & 0.95 \\
\hline $\begin{array}{l}\text { Condition } \\
\text { factor -SL }\end{array}$ & $\begin{array}{c}(\mathrm{BW} \\
\times 10^{3} / \\
\left.\mathrm{SL}^{3}\right)\end{array}$ & 33.6 & \pm & 0.88 & 33.7 & \pm & 1.29 \\
\hline Wi/SL & - & 0.179 & \pm & 0.008 & 0.178 & \pm & 0.008 \\
\hline BD/SL & - & 0.466 & \pm & 0.006 & 0.474 & \pm & 0.010 \\
\hline DCP/SL & - & 0.118 & \pm & 0.003 & 0.118 & \pm & 0.003 \\
\hline
\end{tabular}

Mean \pm Standard deviation. dpf: days post fertilization. N: number of specimens. 


\section{Discussion}

In this study, to estimate the function of Mstnb in growth and muscle development of red seabream, we established the null-allelic mutant of Pm-mstnb from spontaneous mating of genetically-mosaic Pm-mstnb-edited broodstocks which had been created by CRISPR/Cas 9 system. To establish the nullallelic mutant, we first assessed the soma mutation of $\mathrm{G}_{0}$. As a result, even the soma of $\mathrm{G}_{0}$ showed genetic mosaicism. The represented variants found in the soma of $\mathrm{G}_{0}$ were also found in $F_{1}$. Strictly speaking, in order to predict the genotype of offspring, the genotype of the parents' germ cells should be examined, especially in the case of geneticallymosaic parents. However, our results suggest that, even without examining parental germline mutations, knowing the parent's somatic mutations would also be helpful for predicting the genotype of the next generation of individuals. After rearing $F_{1}$, we compared the growth traits and body shape of Pm-mstnb ${ }^{w t / w t}$ and Pm-mstn $b^{-5 /-5}$. However, Pm$m s t n b^{-5 /-5}$ showed no significant differences in terms of the growth traits and the body shape from that of Pm-mstn $b^{w t / w t}$. Here, we discuss the biological function of Mstnb in red seabream in relation to duplicated genes, the relatedness between species and other possibilities.

Unlike mammals, most of the teleost fish have duplicated $m s t n$, which were thought have varied functions in some tissues and shared functions in others (De Santis and Jerry, 2011; Helterline et al., 2007; Wang et al., 2018; Xie et al., 2019). For example, in deleterious mutants of zebrafish, while the deficiency of one paralog mstn showed the increased muscle phenotype, the deficiency of the other mstn paralog had no apparent phenotype in terms of muscle development and growth (Wang et al., 2018). It was also reported that both the paralogs were expressed in the spleen when fish were stressed, and both were involved in the zebrafish immune system (Helterline et al., 2007; Wang et al., 2018). Although this functional sharing was assumed between the mstn paralogs, either one could play the main role as a negative regulator of muscle growth. Red seabream also has two mstn candidates as a negative regulator of skeletal muscle mass (Kishimoto et al., 2018). In a previous study, we had demonstrated that the deficiency of Pm-mstn causes increased muscle mass, meaning an edible part, with changing body shape (Kishimoto et al., 2018). These Pmmstn deficient fish had higher condition factor and increased body width and depth as a result of increasing muscle, and also showed a shortened body length and miniaturization of the vertebra, compared to those of wild type fish (Kishimoto et al., 2018). The previous study also showed that the $\mathrm{G}_{0}$ of Pm-mstnb disrupted line represented no apparent muscular phenotype (Kishimoto et al., 2018). However, because the first generation $\left(\mathrm{G}_{0}\right)$ in which gene-knockout mutations are introduced through CRISPR/Cas9 zygote microinjection shows genetic mosaicism (Mehravar et al., 2019), comparisons between $\mathrm{G}_{0}$ gene-edited and wild-type organisms have limited utility to detect the effects of the gene knockout. We thought the potential for Pm-mstnb to be involved in muscle development may have been hidden by genetic mosaicism in the Pm-mstnb $\mathrm{G}_{0}$. Therefore, to more fully assess the role of Pm-mstnb in muscle development and body mass growth in this study, we created Pm-mstnb nullallelic fish. In the case of Pm-mstnb null-allelic young-fish, there were no significant differences in terms of growth traits and body shape compared to that of the wild type. These results supported the hypothesis that the Pm-mstnb does not regulate body growth and muscle development in young red seabream. To confirm this hypothesis, follow-up experiments should be conducted to 1) to determine through histological observation that muscle fiber growth has not changed in the Pm-mstnb mutants, 2) to confirm that the $m s t n b$ mutants were truly deficient in the Mstnb protein, and 3) to examine how spontaneous the expression of Pm-mstnb in the muscular tissue of the wild type was.

We also supposed that the functional sharing states of Pm-mstn paralogues in red seabream may resemble those of zebrafish, wherein one gene acts as a negative muscle regulator, whereas the other one does not and is of unknown function. Our results supported this. These trends of the functional sharing states of mstn paralogues might be common in the teleost because the paralogous-mutants in two genetically divergent species (red seabream as a family of Perciformes and zebrafish as a family of Cypriniformes) showed the resemble phenotypes. Like other species of aquaculture-targeted fish, red seabream has a bigger body size and a longer life span than zebrafish and keeps growing until death. It is still possible that these functional sharing states of Pm-mstn paralogues might change over the course of their growing and aging events, such as spawning, starving, and contagion.

In general, TGF- $\beta$ superfamilies including Mstn are thought to have the varied functions, such as proliferation, differentiation and apoptosis of cells in the varied organs such as the brain, internal organs, reproductive organ and muscle (Kashima and Hata, 2018; Phelps et al., 2013; Zhang et al., 2017; Zheng et al., 2018). To further explore unknown functions of the two Pm-mstn paralogues, both of the nullallelic mutants should be tested with regards to their possible functions in the immune system, sexual maturation, behaviors and so on.

Although our results were preliminary because the analyzed number of fish and the analyzed stage were small and limited, respectively, we presumed that Pm-mstnb is not involved in the body growth and the muscle growth in young red seabream. We conclude that, for improving the processing yield by increasing the edible part of red seabream, Pm-mstn is a more feasible target for mutation than Pm-mstnb. The homology and conserved functions in paralogs of these genes between divergent fish species suggests there is considerable potential to apply targeted DNA editing to Mstn paralogs in other finfish species. Finally, we suggest to find the Mstn paralog, which bring muscle mass increasing trait, among Mstn paralogs in the genome of aquaculture fish. As we have shown in a previous paper (Kishimoto et al., 2018), it is important to create the 
phylogenetic tree of the multispecies from the gene sequences of Mstn paralogs, and then select certain paralog belongs to the same clade to Pm-mstn. To distinguish certain paralog, it is also important to ensure that the orthologous relationship is conserved to Pm-mstn, with synteny analysis using genomic sequences and gene information around Mstn paralogs of the target fish.

\section{Acknowledgement}

The authors would like to thank Atsushi TOYODA for computational assistance with the deep sequencing. We also thank Satoshi ANSAI for lending his expertise on the genome editing. We would like to thank Editage (www.editage.com) for English language editing. Finally, we are grateful to the referees and editors for useful comments. This work was supported by the Grant-in-Aid for Scientific Research (B) 26292104 (M.K. and Keitaro K.), Japan.

\section{Author contributions}

The contributions are as follows. Conceptualization: M.K., Keitaro K. and Y.W. Rearing fish: M.O., Keitaro K. and Y.W. Data curation and formal analysis: M.O., Kenta K. and Y.W. Funding acquisition: M.K. and Keitaro K. Investigation: M.O. and Y.W. Methodology: all authors. Project administration: M.K., Keitaro K. and Y.W. Supervision: Y.W. Validation: M.K., Keitaro K. and Y.W. Visualization: M.O. and Y.W. Writing original draft: M.O. and Y.W. Writing - review \& editing: Kenta K., M.K., Keitaro K. and Y.W.

\section{Competing Financial Interests}

The authors declare no conflicts of interest associated with this manuscript.

\section{References} Ansai, S., Kinoshita, M. (2014). Targeted mutagenesis using CRISPR/Cas
system in medaka. Biology Open 3, $362-371$.
https://doi.org/10.1242/bio.20148177

Brocal, I., White, R.J., Dooley, C.M., Carruthers, S.N., Clark, R., Hall, A., Busch-Nentwich, E.M., Stemple, D.L., Kettleborough, R.N.W. (2016). Efficient identification of CRISPR/Cas9-induced insertions/deletions by direct germline screening in zebrafish. BMC Genomics 17, 259. https://doi.org/10.1186/s12864-016-2563-z

De Santis, C., Jerry, D.R. (2011). Differential tissue-regulation of myostatin genes in the teleost fish Lates calcarifer in response to fasting. Evidence for functional differentiation. Molecular and Cellular Endocrinology 335, 158-165. https://doi.org/10.1016/j.mce.2011.01.011

Gratacap, R.L., Wargelius, A., Edvardsen, R.B., Houston, R.D. (2019). Potential of genome editing to improve aquaculture breeding and production. Trends in Genetics 35, 672-684. https://doi.org/10.1016/j.tig.2019.06.006

Helterline, D.L.I., Garikipati, D., Stenkamp, D.L., Rodgers, B.D. (2007). Embryonic and tissue-specific regulation of myostatin-1 and -2 gene expression in zebrafish. General and Comparative Endocrinology 151, 9097. https://doi.org/10.1016/j.ygcen.2006.12.023

Kashima, R., Hata, A. (2018). The role of TGF- $\beta$ superfamily signaling in neurological disorders. Acta Biochimica et Biophysica Sinica 50, 106-120. https://doi.org/10.1093/abbs/gmx124
Kishimoto, K., Washio, Y., Murakami, Y., Katayama, T., Kuroyanagi, M., Kato, K., Yoshiura, Y., Kinoshita, M. (2019). An effective microinjection method for genome editing of marine aquaculture fish: tiger pufferfish Takifugu rubripes and red sea bream Pagrus major. Fisheries Science 85, 217-226. https://doi.org/10.1007/s12562-018-1277-3

Kishimoto, K., Washio, Y., Yoshiura, Y., Toyoda, A., Ueno, T., Fukuyama, H., Kato, K., Kinoshita, M. (2018). Production of a breed of red sea bream Pagrus major with an increase of skeletal muscle mass and reduced body length by genome editing with CRISPR/Cas9. Aquaculture 495, 415-427. https://doi.org/10.1016/j.aquaculture.2018.05.055

Mehravar, M., Shirazi, A., Nazari, M., Banan, M. (2019). Mosaicism in CRISPR/Cas9-mediated genome editing. Developmental Biology 445, 156 162. https://doi.org/10.1016/j.ydbio.2018.10.008

Murata, O., Harada, T., Miyashita, S., Izumi, K., Maeda, S., Kato, K., Kumai, H. (1996). Selective Breeding for Growth in Red Sea Bream. Fisheries science 62, 845-849. https://doi.org/10.2331/fishsci.62.845

Okauchi, M. (2004). An assessment of the beneficial roles of Nannochloropsis oculata in larval rearing of marine finfish. BulletinFisheries Research Agency Japan 8, 83-90.

Phelps, M.P., Jaffe, I.M., Bradley, T.M. (2013). Muscle growth in teleost fish is regulated by factors utilizing the activin II B receptor. Journal of $\begin{array}{llll}\text { Experimental } & \text { Biology } & 216, & 3742-3750\end{array}$ https://doi.org/10.1242/jeb.086660

Tait-Burkard, C., Doeschl-Wilson, A., McGrew, M.J., Archibald, A.L., Sang H.M., Houston, R.D., Whitelaw, C.B., Watson, M. (2018). Livestock $2.0-$ genome editing for fitter, healthier, and more productive farmed animals. Genome Biology 19, 204. https://doi.org/10.1186/s13059-018-1583-1

Wang, C., Chen, Y.-L., Bian, W.-P., Xie, S.-L., Qi, G.-L., Liu, L., Strauss, P.R., Zou, J.-X., Pei, D.-S. (2018). Deletion of mstna and mstnb impairs the immune system and affects growth performance in zebrafish. Fish and Shellfish Immunology 72, 572-580. https://doi.org/10.1016/j.fsi.2017.11.040

Xie, S., Zhou, A., Feng, Y., Wang, Z., Fan, L., Zhang, Y., Zeng, F., Zou, J. (2019). Effects of fasting and re-feeding on mstn and mstnb genes expressions in Cranoglanis bouderius. Gene 682, 1-12. https://doi.org/10.1016/j.gene.2018.09.050

Xiong, J.-S., Ding, J., Li, Y. (2015). Genome-editing technologies and their potential application in horticultural crop breeding. Horticulture Research 2, 15019. https://doi.org/10.1038/hortres.2015.19

Zhang, Y., Alexander, P.B., Wang, X.-F. (2017). TGF- $\beta$ Family Signaling in the Control of Cell Proliferation and Survival. Cold Spring Harbor $\begin{array}{llll}\text { Perspectives in Biology 9022145. } & \text { 9, }\end{array}$ https://doi.org/10.1101/cshperspect.a022145

Zheng, S., Long, J., Liu, Z., Tao, W., Wang, D. (2018). Identification and Evolution of TGF- $\beta$ Signaling Pathway Members in Twenty-Four Animal Species and Expression in Tilapia. International Journal of Molecular Sciences 19, 1154. https://doi.org/10.3390/ijms19041154 